\title{
ANALYSIS OF SCATTERING COMPONENTS FROM FULLY POLARIMETRIC SAR IMAGES FOR IMPROVING ACCURACIES OF URBAN DENSITY ESTIMATION
}

\author{
J. Susaki* \\ Department of Civil and Earth Resources Engineering, Graduate School of Engineering, Kyoto University, Japan \\ susaki.junichi.3r@kyoto-u.ac.jp
}

Commission VII, WG VII/1

KEY WORDS: Urban scattering, fully polarimetric SAR, probability density function, normalization of scattering, four component decomposition

\begin{abstract}
:
In this paper, we analyze probability density functions (PDFs) of scatterings derived from fully polarimetric synthetic aperture radar (SAR) images for improving the accuracies of estimated urban density. We have reported a method for estimating urban density that uses an index $T_{v+c}$ obtained by normalizing the sum of volume and helix scatterings $P_{v+c}$. Validation results showed that estimated urban densities have a high correlation with building-to-land ratios (Kajimoto and Susaki, 2013b; Susaki et al., 2014). While the method is found to be effective for estimating urban density, it is not clear why $T_{v+c}$ is more effective than indices derived from other scatterings, such as surface or double-bounce scatterings, observed in urban areas. In this research, we focus on PDFs of scatterings derived from fully polarimetric SAR images in terms of scattering normalization. First, we introduce a theoretical PDF that assumes that image pixels have scatterers showing random backscattering. We then generate PDFs of scatterings derived from observations of concrete blocks with different orientation angles, and from a satellite-based fully polarimetric SAR image. The analysis of the PDFs and the derived statistics reveals that the curves of the PDFs of $P_{v+c}$ are the most similar to the normal distribution among all the scatterings derived from fully polarimetric SAR images. It was found that $T_{v+c}$ works most effectively because of its similarity to the normal distribution.
\end{abstract}

\section{INTRODUCTION}

Land cover maps are one of the most useful products derived from remotely sensed images. Urban area maps, in particular, are valuable in understanding the status of human activities and discussing future directions of economic growth. Synthetic aperture radar (SAR) is capable of generating urban maps because it can detect backscattering from terrestrial objects, including artifacts in urban areas. Some urban area classifiers using SAR images have been proposed (Niu and Ban, 2012; Perissin and Wang, 2012; Esch et al. 2013).

To accurately extract urban areas and generate value-added thematic maps from SAR images, an understanding of microwave surface scattering is indispensable. Polarimetric radar allows for more advanced microwave scattering models. The scattering matrix measured by polarimetric radar represents the complex process of electromagnetic interaction phenomena between the target structure and the emitted electromagnetic field (Lee and Pottier, 2009). Target decomposition approaches have been investigated to derive information from the objects of interest, and two approaches have been widely applied. One is to use an eigenvector or eigenvalues derived from covariance or coherency matrices (Cloude and Pottier, 1997). These matrices are generated from the originally obtained scattering matrix. The other is to apply a "model-based" decomposition of the covariance matrix or coherency matrix (Freeman and Durden, 1998; Yamaguchi et al., 2006). This approach assumes fundamental scatterings, such as surface scattering, double-bounce scattering, or volume scattering. Following the scattering properties, each scattering is expressed as a scattering matrix. Finally, the contribution of each scattering is determined by solving equations under several parameter assumptions. This model-based approach contributes to

${ }^{*}$ Corresponding author. understanding land surfaces in terms of their physical scattering properties.

In 2006, the Japan Aerospace Exploration Agency (JAXA) launched the first satellite-borne fully polarimetric SAR (PolSAR) sensor, the Advanced Land Observing Satellite (ALOS) / Phased Array type L-band SAR (PALSAR). This success promoted research using PolSAR images. From these data, urban areas are easily extracted by lessening the effects of the orientation angles of objects against the radar beam (Kajimoto and Susaki, 2013a). The method utilizes volumetric scattering derived from four-component decomposition methods (Yamaguchi et al., 2011). In addition, a method was presented that estimates urban density from a fully PolSAR image (Kajimoto and Susaki, 2013b; Susaki et al., 2014). The method uses an index $T_{v+c}$ obtained by normalizing the sum of volume and helix scatterings $P_{v+c}$. Volume and helix scatterings are generated by applying the four-component decomposition method (Yamaguchi et al., 2011) to a fully PolSAR image. The validation results show that estimated urban densities have a high correlation with building-to-land ratios. However, buildings return stronger surface or double-bounce scatterings than volumetric or helix scatterings. It remains unclear why $T_{v+c}$ is more effective than indices derived from surface or doublebounce scatterings.

Another issue is that the proposed method may overestimate urban densities of areas in which the polarimetric orientation angles (POAs) are almost $0^{\circ}$ and strong backscattering is observed. The method normalizes $P_{v+c}$ by using the mean and standard deviation, thus lessening the POA effects. In reality, this approach may not remove all POA effects, as described in Section 2. The normalization implicitly assumes that the probability density function (PDF) of the scattering indicates a normal distribution. However, it has been not confirmed whether this is the case in PDFs of the scattering derived from actual images. 
In this paper, we analyze PDFs of scatterings derived from fully PolSAR images for improving the accuracies of estimated urban density. The remainder of this paper is organized as follows. Our previous work related to estimating urban density and the method is described in Section 2. After a theoretical PDF of scattering is introduced in Section 3, the statistical characteristics of scattering obtained by the experiments are analyzed in Section 4. The implications of these results are then discussed in Section 5. Finally, Section 6 concludes the paper.

\section{PREVIOUS WORK}

\subsection{Data Used}

A previous study (Susaki et al., 2014) used fully polarimetric ALOS/PALSAR level 1.1 (L1.1) data. In this paper, we report the results of using data for New York City. The observation date was April 1, 2011. The image has slant-range coordinate data and a $21.5^{\circ}$ off-nadir angle against the center of the scene. In addition, ALOS/Advanced Visible and Near Infrared Radiometer type-2 (AVNIR-2) optical sensor data were used as a reference.

\subsection{Estimated Urban Density}

The method for estimating urban density was as follows. We extracted urban areas by applying the method proposed by (Kajimoto and Susaki, 2013a). Then, we applied a four component decomposition method that corrects POA angle effects (Yamaguchi et al., 2011). It was reported that a normalized combination of the volume scattering power and the helix scattering power $\left(T_{v+c}\right)$ shows the best correlation with both the building-to-land ratio and the floor area ratio (Equation (1) (Kajimoto and Susaki, 2013b). The normalization uses mean and standard deviation of the volume scattering power and the helix scattering power $\left(P_{v+c}\right)$. To compare the status of urban development of global megacities, we extended the method that the mean $\mu$ and standard deviation $\sigma$ should be obtained via all study areas (Equations (2) and (3)).

$$
\begin{aligned}
T_{v+c}(i, j, k)= & \frac{P_{v+c}(i, j, k, \phi, H)-\mu_{v+c}(\phi, H)}{\sigma_{v+c}(\phi, H)} \\
\mu_{v+c}(\phi, H)= & \frac{1}{N(\phi, H)} \sum_{i} \sum_{j} \sum_{k} P_{v+c}(i, j, k, \phi, H) \\
\sigma_{v+c}^{2}(\phi, H)= & \frac{1}{N(\phi, H)} \sum_{i} \sum_{j} \sum_{k} \\
& \left(P_{v+c}(i, j, k, \phi, H)-\mu_{v+c}(\phi, H)\right)^{2}
\end{aligned}
$$

Here, $(i, j)$ is the localtion of the reference pixel, $k$ is the SAR image number, $\phi$ is POA, $H$ is the POA type (homogeneous or heterogeneous POA) and $N$ is the number of pixels.

Figure 1 shows the estimated urban density of New York. The areas in a circle and an ellipse in Fig. 1(b) have relatively lowstoried buildings according to other reference data (Open Street Map, 2014). Because the orientation angles of the buildings against radar are almost $0^{\circ}$, the total powers (TPs) observed in these areas are much higher than in nearby areas. Hereafter, we refer to these areas as "high-TP areas." Actual building-to-land ratios of the high- $T P$ and nearby areas are similar. In Fig. 1(d), the contrasts of indices between the high- $T P$ areas and the nearby areas were lessened, but the high-TP areas still have higher values. Validation using the reference data confirmed that the urban densities of high- $T P$ areas are overestimated.

Equation (1) normalizes $P_{v+c}$ by using the mean and standard deviation. It is assumed that the PDF of $P_{v+c}$ should follow a normal distribution. Following the findings obtained from Fig. 1, our motivations are to examine the PDFs of $P_{v+c}$ and other scattering components for improving the accuracies of estimated urban densities.

\section{STATISTICAL FEATURES OF SAR IMAGES}

Before examining actual PDFs of scattering obtained from images, we derive the theoretical PDF of scattering.

\subsection{Amplitude and Phase}

We assume that a pixel in an SAR image has scatterers, with the amplitude and phase of scatterer $i$ expressed as $A_{i}$ and $\phi_{i}$, respectively. The observed data of a pixel is expressed by Eq. (4):

$$
V_{R}+i V_{I}=\sum_{i} A_{i} \exp \left(i \phi_{i}\right)
$$

Here, $V_{R}$ and $V_{I}$ are the real and imaginary parts of the observed data, respectively. Because $V_{R}$ and $V_{I}$ are sum of random variables, the PDFs of $V_{R}$ and $V_{I}$ follow a normal distribution.

$$
\begin{aligned}
& p\left(V_{R}\right)=\frac{1}{\sqrt{2 \pi} \sigma} \exp \left(-\frac{V_{R}^{2}}{2 \sigma^{2}}\right), \\
& p\left(V_{I}\right)=\frac{1}{\sqrt{2 \pi} \sigma} \exp \left(-\frac{V_{I}^{2}}{2 \sigma^{2}}\right) .
\end{aligned}
$$

In addition, we assume that the means of $V_{R}$ and $V_{I}$ are equal to 0 , the variances are equal to $\sigma^{2}$, and $V_{R}$ and $V_{I}$ are independent.

$$
E\left[V_{R} V_{I}\right]=E\left[V_{R}\right] E\left[V_{I}\right]=0 .
$$

The joint PDF of $V_{R}$ and $V_{I}, p\left(V_{R}, V_{I}\right)$, is expressed by Equation (8).

$$
p\left(V_{R}, V_{I}\right)=\frac{1}{2 \pi \sigma^{2}} \exp \left(-\frac{V_{R}^{2}+V_{I}^{2}}{2 \sigma^{2}}\right) .
$$

Equation (8) is rewritten using amplitude $A$ and phase $\phi$ of the pixel data as

$$
p(A, \phi)=\frac{A}{2 \pi \sigma^{2}} \exp \left(-\frac{A^{2}}{2 \sigma^{2}}\right) .
$$

Here, $A=\sqrt{V_{R}^{2}+V_{I}^{2}}$.

The PDFs $p(A)$ and $p(\phi)$ are obtained by integrating Equation (9) in terms of $\phi$ and $A$, respectively:

$$
\begin{aligned}
& p(A)=\int_{0}^{2 \pi} p(A, \phi) d \phi=\frac{A}{\sigma^{2}} \exp \left(-\frac{A^{2}}{2 \sigma^{2}}\right), \\
& p(\phi)=\int_{0}^{\infty} p(A, \phi) d A=\frac{1}{2 \pi} .
\end{aligned}
$$

It is shown that $p(A)$ and $p(\phi)$ follow Rayleigh and uniform distributions, respectively.

\subsection{Power}

We assume the power $I=A^{2}$, and derive the PDF $p(I)$ as

$$
p(I)=\frac{1}{2 \sigma^{2}} \exp \left(-\frac{I}{2 \sigma^{2}}\right) .
$$

Equation (12) shows that $p(I)$ follows an exponential distribution. 


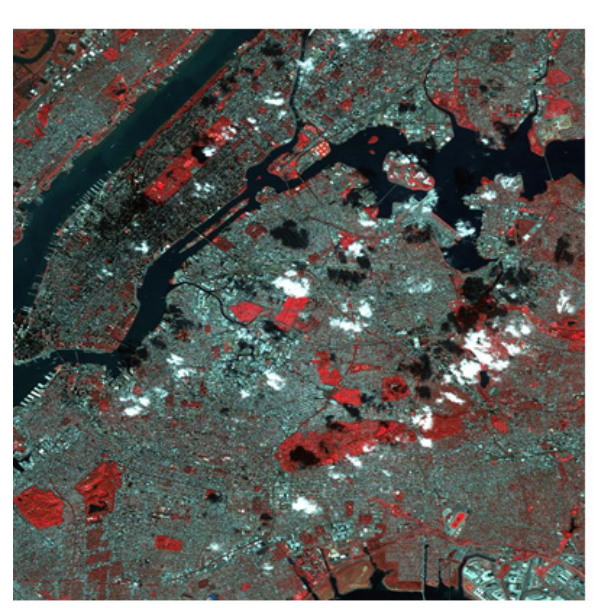

(a)

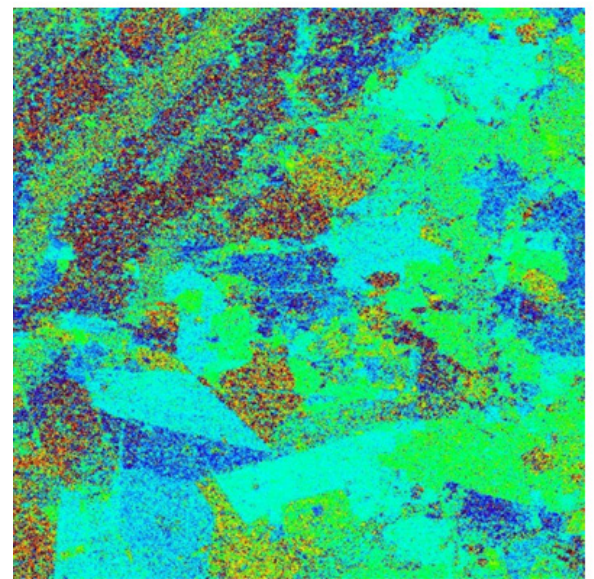

(c)

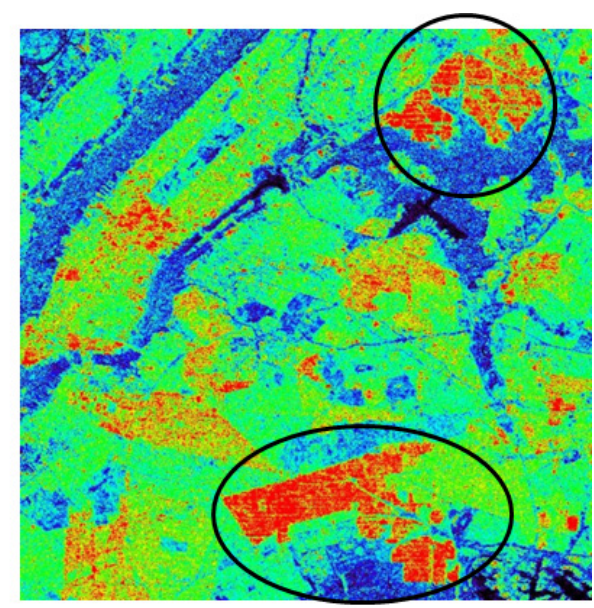

(b)

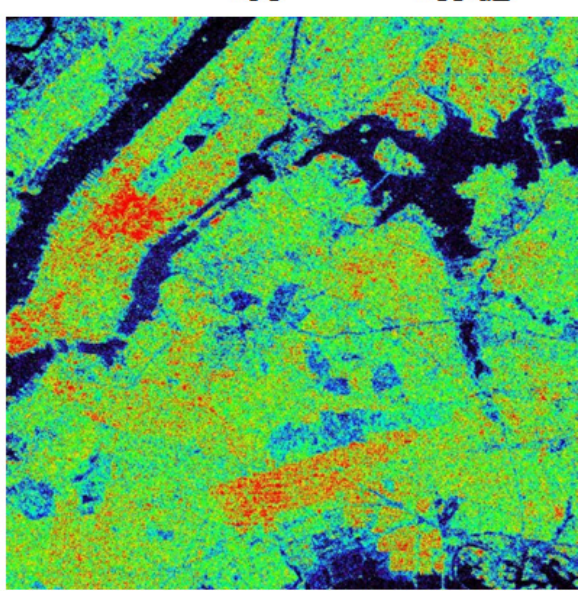

(d)

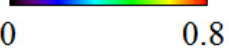

Figure 1. Urban density of New York estimated from a fully polarimetric PALSAR image. (a) AVNIR-2 image, (b) TP, (c) POA and (d) $T_{v+c}$. In the circle and ellipse of (b), where low-storied buildings are available, strong backscattering is observed because the POAs are almost $0^{\circ}$. In (d), $T_{v+c}$ was calculated in the urban areas estimated by applying the method reported by Kajimoto and Susaki (2013a). Each panel has a slant range coordinate system.

In the processing of SAR images, power is converted into decibel (dB) by Equation (13):

$$
I_{\mathrm{dB}}=10 \log _{10} I .
$$

Here, $I_{\mathrm{dB}}$ has units of $\mathrm{dB}$. The PDF $p\left(I_{\mathrm{dB}}\right)$ is expressed by Equation (14).

$$
p\left(I_{\mathrm{dB}}\right)=\frac{\ln 10}{20 \sigma^{2}} 10^{I^{\prime} / 10} \cdot \exp \left(-\frac{10^{I^{\prime} / 10}}{2 \sigma^{2}}\right)
$$

Here, $I^{\prime}$ is $I_{\mathrm{dB}}$. Figure 2 shows curves of Equation (14) with different values of $\sigma$. It shows that $p\left(I_{\mathrm{dB}}\right)$ has a non-normal distribution. To quantitatively assess similarity of the PDFs to a normal distribution, we calculate the skew and kurtosis as

$$
\begin{aligned}
\text { skew } & =\frac{E(X-\mu)^{3}}{\sigma^{3}}, \\
\text { kurtosis } & =\frac{E(X-\mu)^{4}}{\sigma^{4}} .
\end{aligned}
$$

Here, $X, \mu$, and $\sigma$ are a variable, the mean, and the standard deviation, respectively. The results are shown in Table 1 . It is known that a PDF of normal distribution has a skew of 0 and kurtosis of 3. Table 1 shows that the PDF expressed by Equation (14) is regarded as a non-normal distribution.

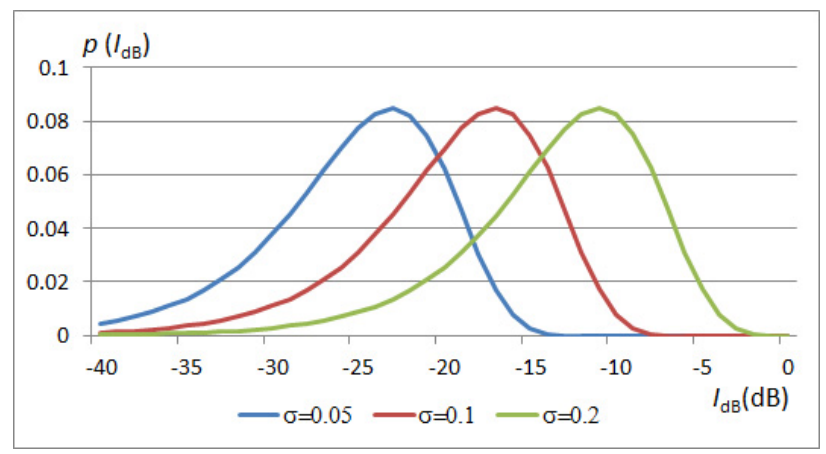

Figure 2. Curves of probability density functions expressed by Equation (14). 


\begin{tabular}{|c|c|c|c|c|}
\hline $\begin{array}{c}\sigma \text { of } \\
\text { Equation (14) }\end{array}$ & mean & $\begin{array}{c}\text { standard } \\
\text { deviation }\end{array}$ & skew & kurtosis - 3 \\
\hline 0.05 & -25.46 & 5.43 & -0.96 & 1.25 \\
0.1 & -19.48 & 5.52 & -1.06 & 1.78 \\
0.2 & -13.47 & 5.55 & -1.11 & 2.10 \\
\hline
\end{tabular}

Table 1. Statistics of probability density functions expressed by Equation (14)

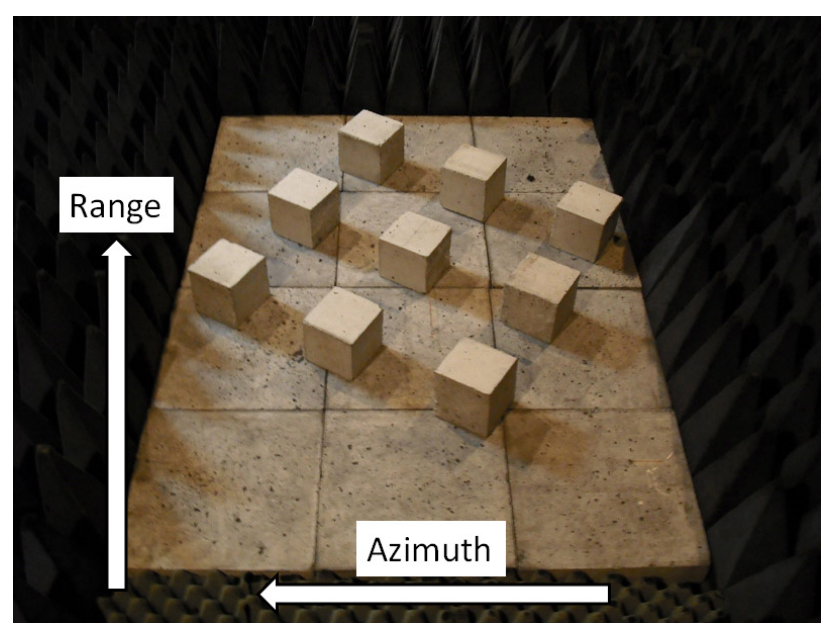

Figure 3. Measurement of scattering of blocks. The distance between the blocks and the orientation angle were $15 \mathrm{~cm}$ and $30^{\circ}$ respectively.

\section{EXPERIMENTS}

\subsection{Data Used}

We used two datasets for analyzing the PDFs of scatterings of urban areas. One is data obtained by an experiment in an anechoic radio wave chamber. The other is a satellite image, ALOS/PALSAR of New York. The details are described in Subsection 2.1.

For the first dataset, obtained on September 29, 2009, an experiment was carried out in the anechoic radio wave chamber at the Wave Engineering Laboratory of the Department of Information Engineering, Faculty of Engineering, Niigata University, Japan. The main purpose of this experiment was to examine how the orientation angle of targets affects the four components of scattering power. A polarimetric measurement system capable of synthetic aperture processing was used. The wave frequency was X-band $(10 \mathrm{GHz})$, antenna height was $260 \mathrm{~cm}$, and the incidence angle was $45^{\circ}$. Nine concrete rectangular blocks with $10 \times 10 \mathrm{~cm}$ base area and $10 \mathrm{~cm}$ height were arranged on a $3 \times 3$ grid. The orientation angle of the target structure is defined as $0^{\circ}$ when the normal to the sides of the blocks is parallel to the sensor's ground range direction. First, measurements were made with the structure at $0^{\circ}$, then the whole structure was rotated clockwise. Measurements were made at orientation angles of $0^{\circ}, 15^{\circ}, 30^{\circ}, 45^{\circ}$, and $60^{\circ}$ with distances of $15 \times 15 \mathrm{~cm}$ between the blocks. Figure 3 shows the blocks when the orientation angle was set to $30^{\circ}$.

\subsection{Results}

Figure 4 shows curves of the scatterings observed from concrete blocks under different orientation angles. Figure 4(a) has curves of $H H, V V, H V$ and $T P$, and Figure 4(b) has those of $P_{s}$, $P_{d}, P_{v}$ and $P_{c}$. Figure 5 shows histograms of scatterings when the orientation angle of the concrete blocks was set to $0^{\circ}$. Figure 5(a) shows histograms of $T P, P_{v}$ and $P_{v+c}$, and Figure 5(b) shows those of $P_{s}$ and $P_{d}$. Similarly, Figures 6,7 and 8 show histograms of scatterings at orientation angles of $15^{\circ}, 30^{\circ}$ and $45^{\circ}$, respectively.

Figure 9 shows histograms of scattering of the areas with POA around $0^{\circ}\left(-2^{\circ} \leq \mathrm{POA} \leq 2^{\circ}\right)$ from PALSAR images of the study area. Kajimoto and Susaki (2013b) reported that the areas should be classified into heterogeneous or homogeneous areas in terms of POA, because they have different histograms of scattering. The histograms of heterogeneous and homogeneous areas are shown in Figure 9. Similarly, Figures 10 and 11 show histograms of scattering in areas with POA around $15^{\circ}\left(13^{\circ} \leq \mathrm{POA} \leq 17^{\circ}\right)$ and $30^{\circ}\left(28^{\circ} \leq \mathrm{POA} \leq 32^{\circ}\right)$, respectively.

The results of the skew and kurtosis of PDFs derived from blocks are shown in Table 2. Those of the study area (New York) derived from an ALOS/PALSAR image are shown in Table 3.

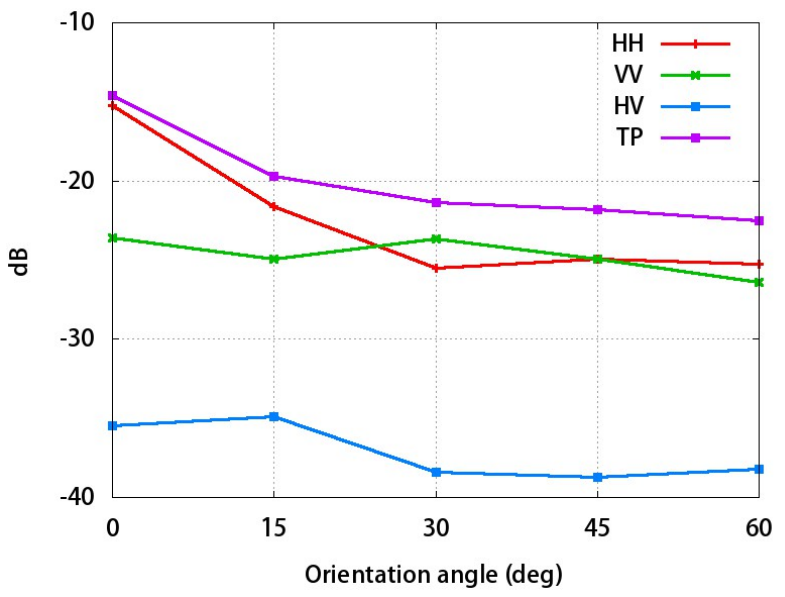

(a)

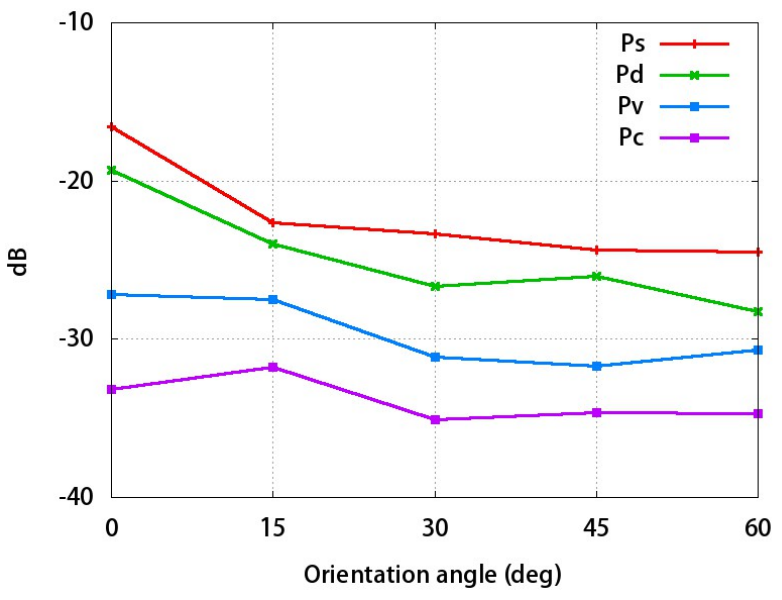

(b)

Figure 4. Scattering of the concrete blocks under different orientation angles. (a) $H H, V V, H V$ and $T P$, and (b) $P_{s}$, $P_{d}, P_{v}$ and $P_{c}$

\section{DISCUSSION}

As explained in Section 1, this research focuses whether the PDF of a scattering is close to a normal distribution from the viewpoint 


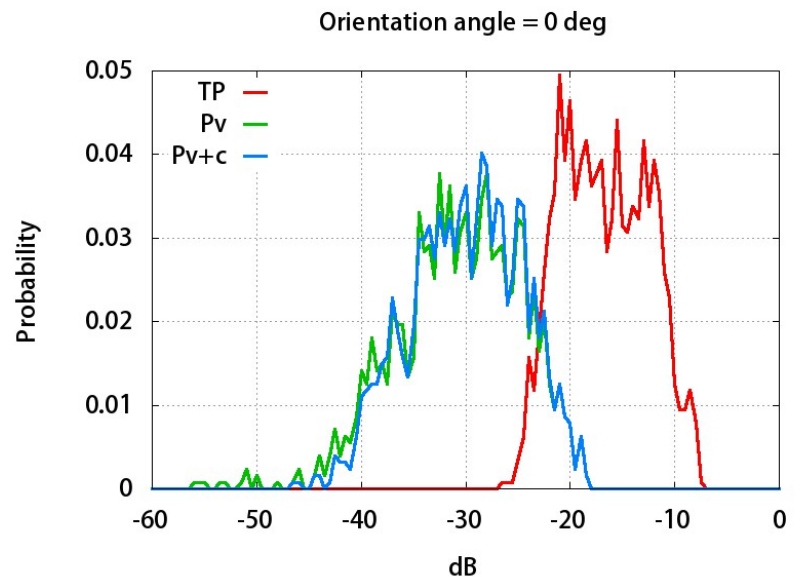

(a)

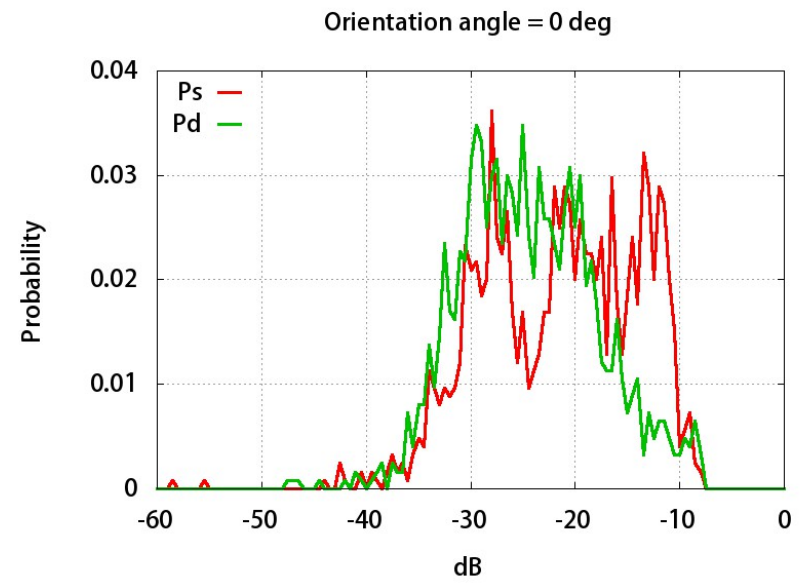

(b)

Figure 5. Histograms of scatterings when the orientation angle of the concrete blocks was set to $0^{\circ}$. (a) $T P, P_{v}$, and $P_{v+c}$, and (b) $P_{s}$ and $P_{d}$

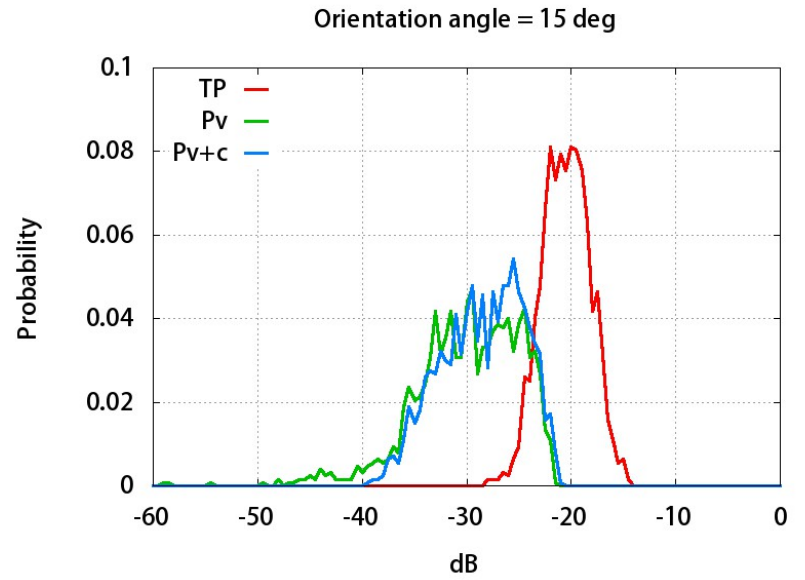

(a)

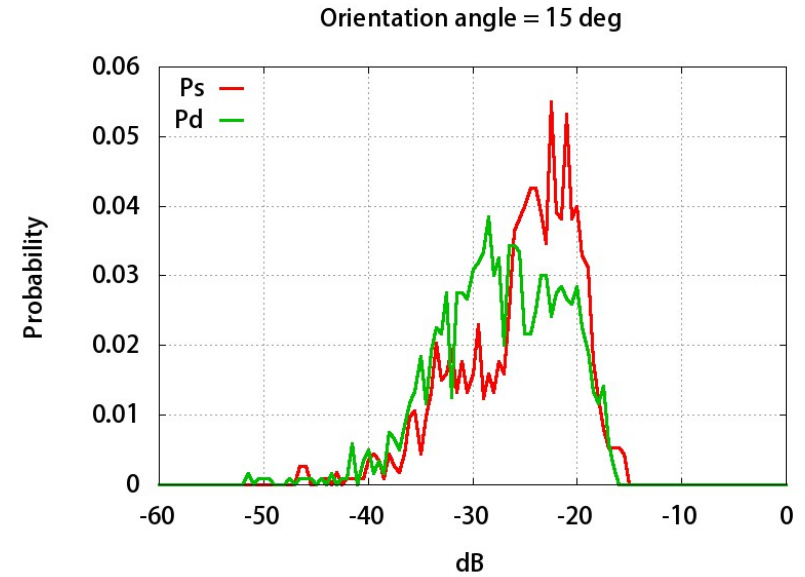

(b)

Figure 6. Histograms of scatterings when the orientation angle of the concrete blocks was set to $15^{\circ}$. (a) $T P, P_{v}$, and $P_{v+c}$, and (b) $P_{s}$ and $P_{d}$

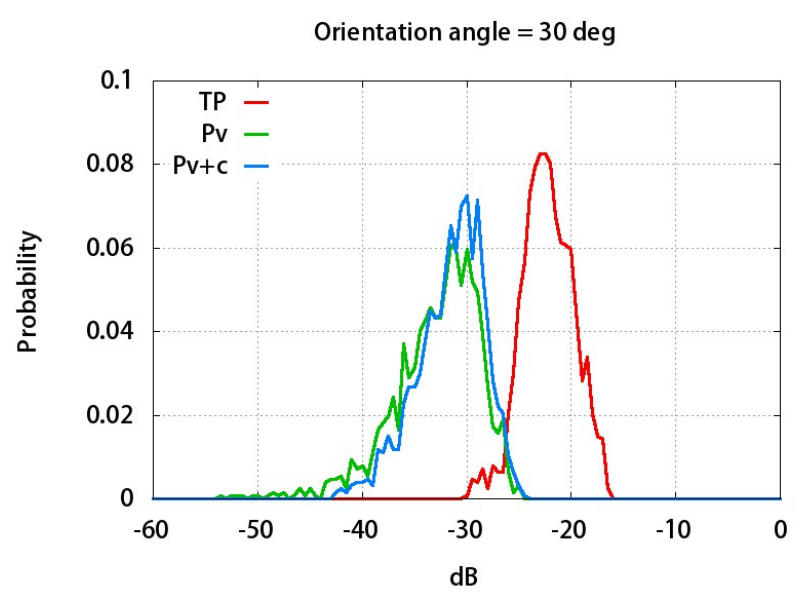

(a)

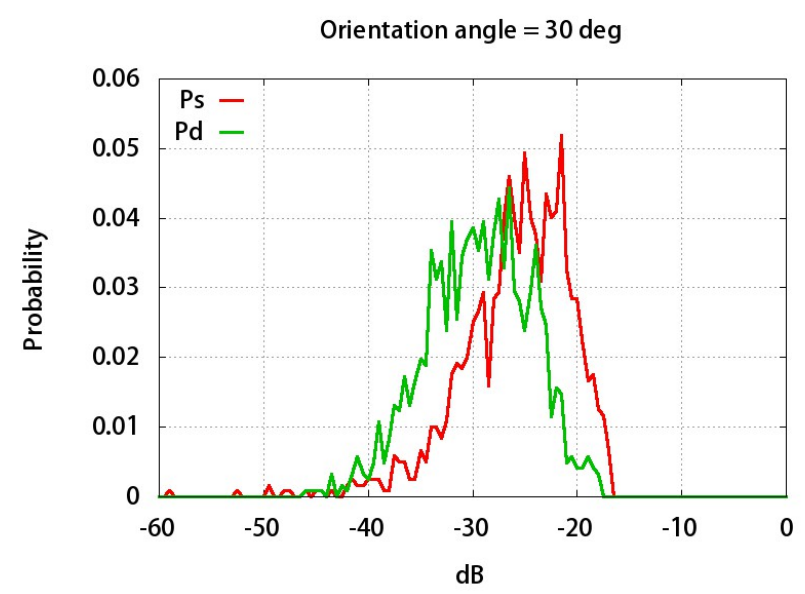

(b)

Figure 7. Histograms of scatterings when the orientation angle of the concrete blocks was set to $30^{\circ}$. (a) $T P, P_{v}$, and $P_{v+c}$, and (b) $P_{s}$ and $P_{d}$ 


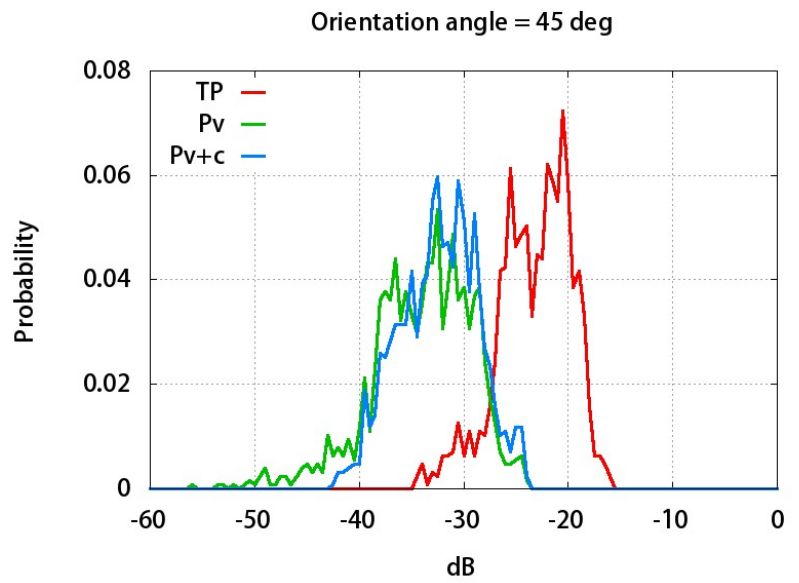

(a)

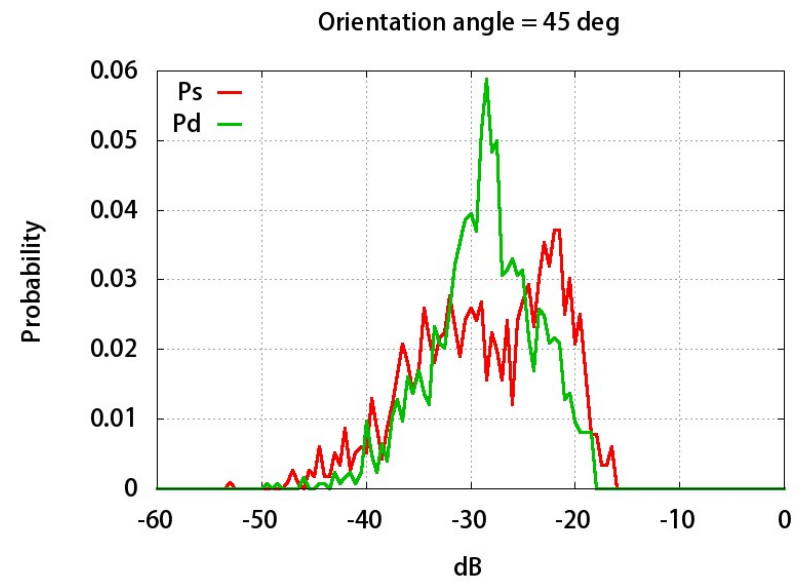

(b)

Figure 8. Histograms of scatterings when the orientation angle of the concrete blocks was set to $45^{\circ}$. (a) $T P, P_{v}$, and $P_{v+c}$, and (b) $P_{s}$ and $P_{d}$

\begin{tabular}{|c|c|c|c|}
\hline POA & Type & skew & kurtosis-3 \\
\hline \multirow{4}{*}{0} & $P_{s}$ & 1.82 & 3.30 \\
& $P_{d}$ & 4.03 & 18.21 \\
& $P_{v}$ & 2.25 & 5.78 \\
& $P_{v+c}$ & 2.21 & 5.63 \\
& $T P$ & 1.67 & 2.82 \\
\hline \multirow{4}{*}{15} & $P_{s}$ & 1.59 & 3.28 \\
& $P_{d}$ & 1.70 & 2.58 \\
& $P_{v}$ & 1.14 & 0.56 \\
& $P_{v+c}$ & 1.07 & 0.53 \\
& $T P$ & 1.28 & 2.09 \\
\hline \multirow{6}{*}{30} & $P_{s}$ & 1.47 & 2.01 \\
& $P_{d}$ & 2.60 & 8.73 \\
& $P_{v}$ & 1.22 & 1.69 \\
& $P_{v+c}$ & 1.09 & 1.40 \\
& $T P$ & 1.27 & 1.42 \\
\hline \multirow{6}{*}{45} & $P_{s}$ & 1.74 & 3.43 \\
& $P_{d}$ & 2.10 & 4.59 \\
& $P_{v}$ & 1.94 & 4.96 \\
& $P_{v+c}$ & 1.85 & 3.99 \\
& $T P$ & 1.07 & 1.22 \\
\hline
\end{tabular}

Table 2. Skew and kurtosis of probability density functions derived from blocks

\begin{tabular}{|c|c|c|c|c|}
\hline POA & Type & Scatter type & skew & kurtosis-3 \\
\hline \multirow{10}{*}{0} & \multirow{5}{*}{ Hetero } & $P_{s}$ & -0.11 & 0.67 \\
\hline & & $P_{d}$ & 0.01 & 0.26 \\
\hline & & $P_{v}$ & -0.93 & 3.08 \\
\hline & & $P_{v+c}$ & 0.21 & 0.10 \\
\hline & & $T P$ & 0.47 & 0.18 \\
\hline & \multirow{5}{*}{ Homo } & $P_{s}$ & -0.44 & 1.08 \\
\hline & & $P_{d}$ & -0.10 & 0.57 \\
\hline & & $P_{v}$ & -1.08 & 3.62 \\
\hline & & $P_{v+c}$ & 0.46 & 0.86 \\
\hline & & $T P$ & 1.04 & 2.02 \\
\hline \multirow{10}{*}{15} & \multirow{5}{*}{ Hetero } & $P_{s}$ & -0.07 & 0.78 \\
\hline & & $P_{d}$ & -0.31 & 1.01 \\
\hline & & $P_{v}$ & -1.06 & 3.06 \\
\hline & & $P_{v+c}$ & 0.25 & 0.73 \\
\hline & & $T P$ & 1.23 & 3.58 \\
\hline & \multirow{5}{*}{ Homo } & $P_{s}$ & -0.60 & 1.18 \\
\hline & & $P_{d}$ & -0.45 & 0.69 \\
\hline & & $P_{v}$ & -1.24 & 4.35 \\
\hline & & $P_{v+c}$ & 0.53 & 1.34 \\
\hline & & $T P$ & 1.11 & 3.73 \\
\hline \multirow{10}{*}{30} & \multirow{5}{*}{ Hetero } & $P_{s}$ & -0.41 & 1.08 \\
\hline & & $P_{d}$ & -0.93 & 0.98 \\
\hline & & $P_{v}$ & -0.37 & 4.35 \\
\hline & & $P_{v+c}$ & 0.94 & 2.59 \\
\hline & & $T P$ & 2.27 & 8.59 \\
\hline & \multirow{5}{*}{ Homo } & $P_{s}$ & -0.73 & 1.49 \\
\hline & & $P_{d}$ & -0.47 & 0.60 \\
\hline & & $P_{v}$ & -1.18 & 4.31 \\
\hline & & $P_{v+c}$ & 0.60 & 1.49 \\
\hline & & $T P$ & 1.13 & 3.68 \\
\hline
\end{tabular}

Table 3. Skew and kurtosis of probability density functions of the study area (New York) derived from an ALOS/PALSAR image 


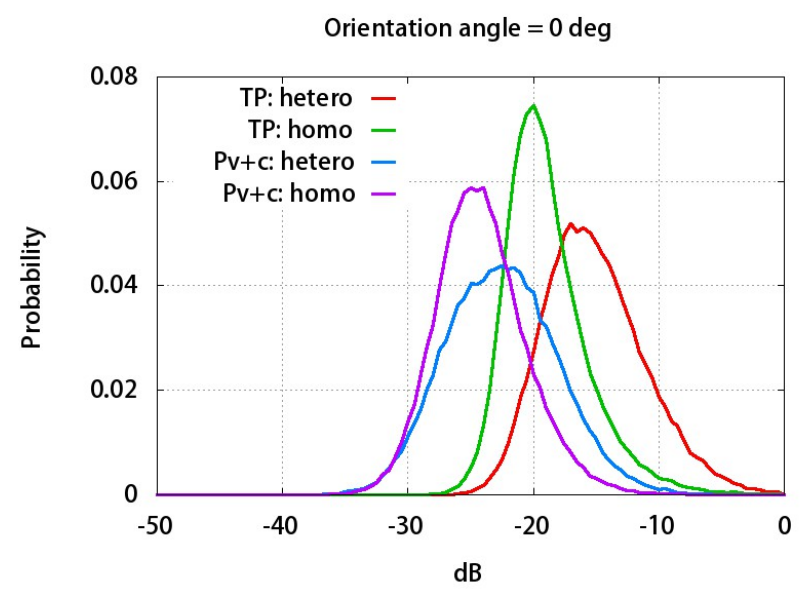

(a)

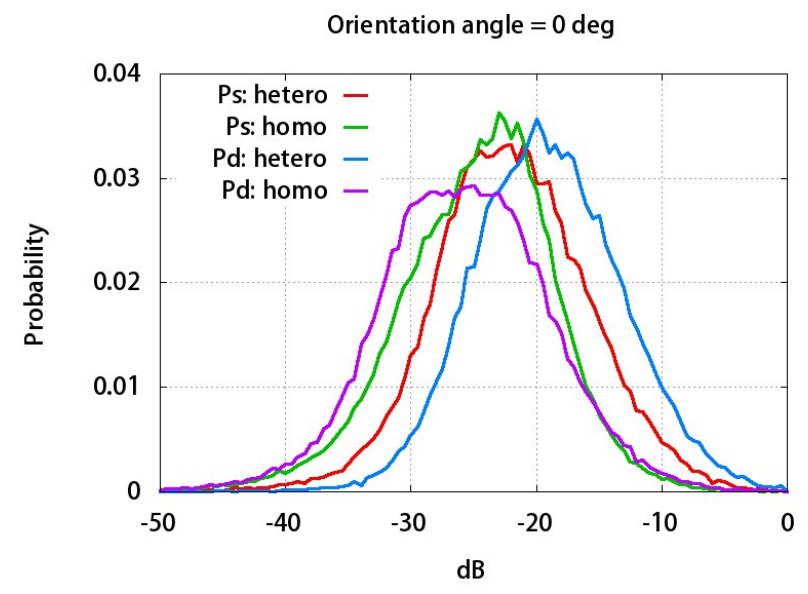

(b)

Figure 9. Histograms of scattering of areas with POA around $0^{\circ}\left(-2^{\circ} \leq \mathrm{POA} \leq 2^{\circ}\right)$ from PALSAR images of the study area. (a) $T P$ and $P_{v+c}$, and (b) $P_{s}$ and $P_{d}$. Here, "hetero" and "homo" denote areas having heterogeneous and homogeneous POA distributions, respectively (Susaki et al., 2014).

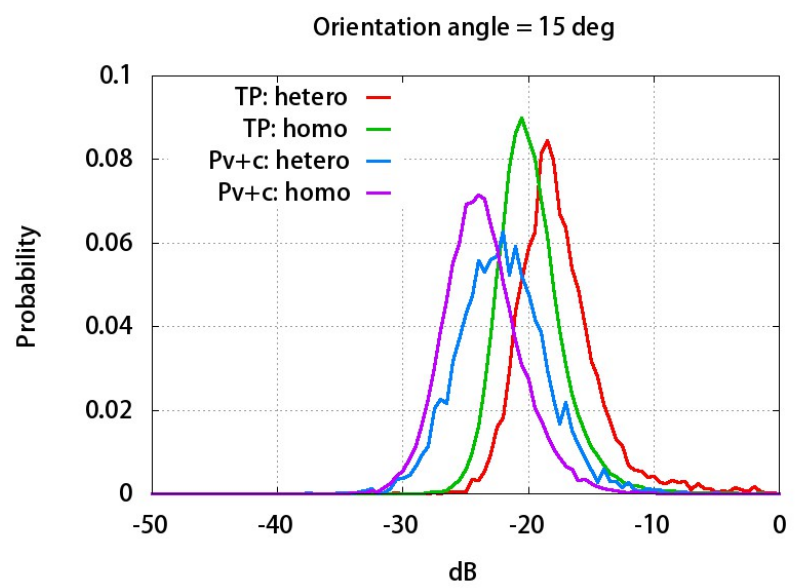

(a)

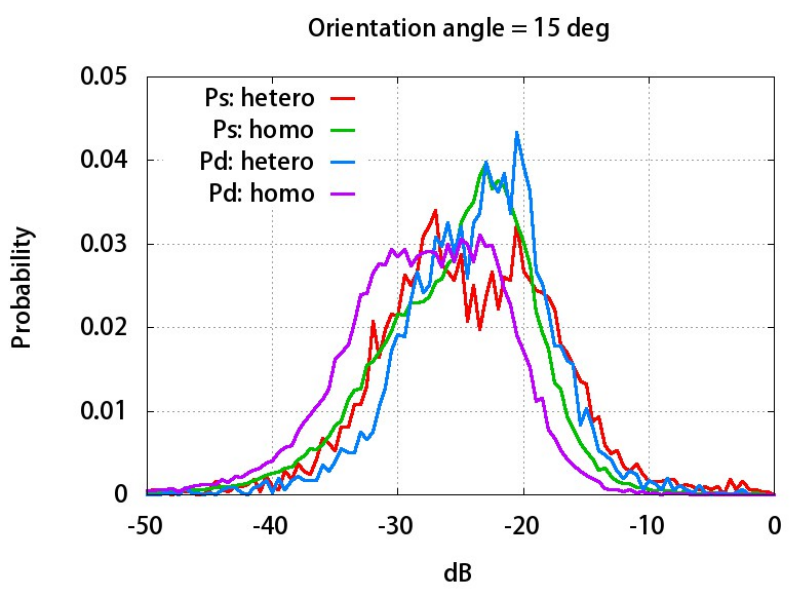

(b)

Figure 10. Histograms of scattering of areas having POA around $15^{\circ}\left(13^{\circ} \leq \mathrm{POA} \leq 17^{\circ}\right.$ from PALSAR image of the study area. See the caption of Fig. 5 .

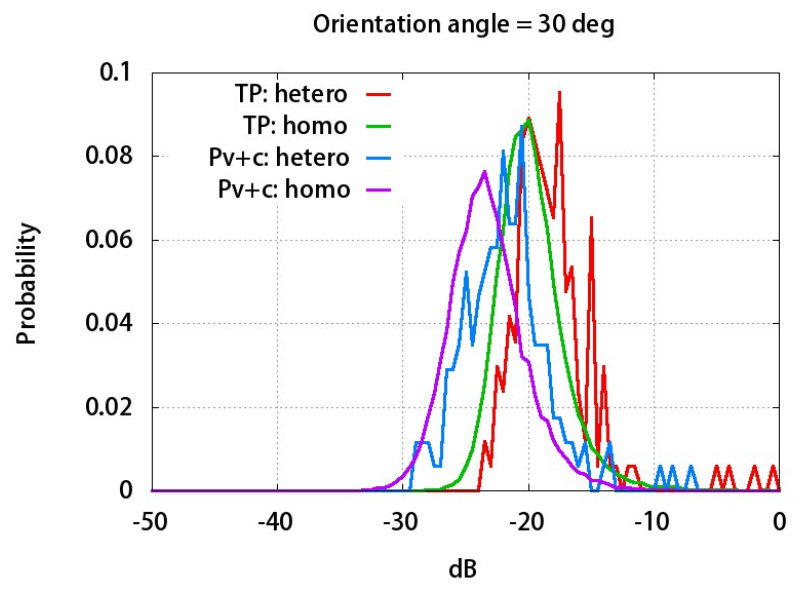

(a)

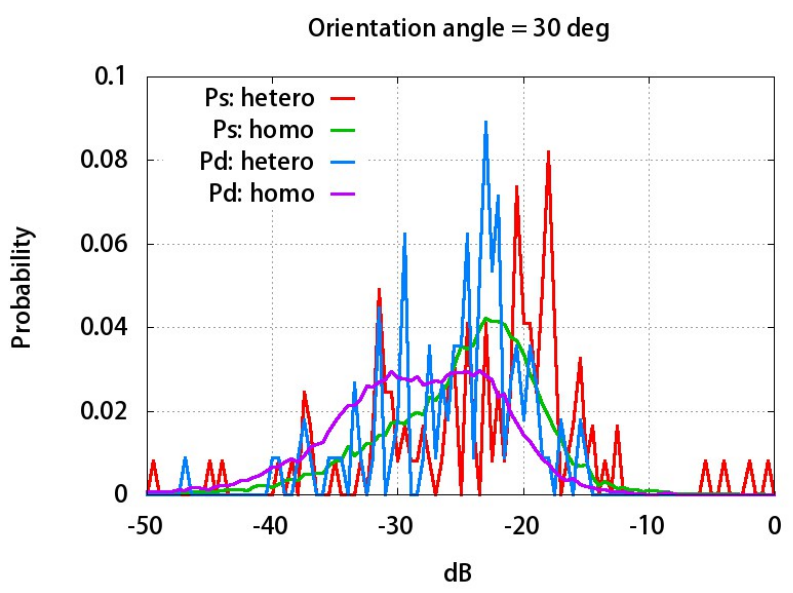

(b)

Figure 11. Histograms of scattering of areas having POA around $30^{\circ}\left(28^{\circ} \leq \mathrm{POA} \leq 32^{\circ}\right)$ from PALSAR image of the study area. See the caption of Fig 5 . 
of scattering normalization. It is difficult to derive definite conclusions from the means of scatterings shown in Figure 4. However, the curves and statistics of PDFs of the scatterings, shown in Figures 5-8 and Table 2 are informative for this purpose. Table 2 shows the skew and the value of kurtosis of PDFs derived from blocks. The smaller the absolute values of the skew and kurtosis - 3 are, the closer to a normal distribution the PDF is. For orientation angles of $15^{\circ}$ and $30^{\circ}, P_{v+c}$ gives the best results for skew and kurtosis. While $P_{v+c}$ has the third best results for orientation angles of $0^{\circ}$ and $45^{\circ}$, it has stable results those are close to the best ones.

Table 3 shows the skew and the value of kurtosis of PDFs derived from an ALOS/PALSAR image. It is similar to the results shown in Table 2, in that $P_{v+c}$ has stable results that are best or close to the best for all POAs. For POA of $30^{\circ}$, Table 3 indicates that $P_{s}$ and $P_{d}$ have the best or similar results. However, Figure 11(b) shows that the curves of $P_{s}$ and $P_{d}$ are not robust. In particular, the curves of $P_{s}$ and $P_{d}$ in heterogeneous areas are sharply fluctuating. On the other hand, the curve of $P_{v+c}$ in heterogeneous areas is the smoothest among all the curves in heterogeneous areas for POA of $30^{\circ}$. Each curve is generated from the same number of samples. It is clear that the smoothness of the curve of PDF depends on scattering type.

According to our previous study (Kajimoto and Susaki, 2013b), $P_{v+c}$ and $P_{v}$ are respectively the best and the second-best scatterings in terms of correlation with building-to-land ratio. For the normalized scattering, $T_{v+c}$ and the normalized $T P$ are the best and second-best scatterings in terms of correlation with buildingto-land ratio, respectively. The results obtained by the experiments in this research are consistent with the results of the previous study. The curves of the PDFs of $P_{v+c}$ are smooth and the most similar to the normal distribution among all the scatterings derived from fully polarimetric SAR images. Because of the similarity to the normal distribution, the normalization of $P_{v+c}$, expressed by Equation (1), works most effectively. Therefore, $T_{v+c}$ is the best scattering for estimating urban density among all the scatterings. However, $P_{v+c}$ is not a normal distribution. To improve the accuracies of estimated urban density, the normalization expressed by Equation (1) should be replaced by another approach that normalizes scatterings without using the mean and standard deviation. In the near future, we will investigate other approaches.

We derived the theoretical PDFs of scatterings, expressed by Equation (14). The experimental results show that each scattering $\left(P_{s}\right.$, $P_{d}, P_{v}, P_{v+c}$, and $T P$ ) has a different function from the theoretical one. This is particularly the case with $P_{v+c}$. These results may indicate that the assumption that a pixel has random scatterers does not apply to actual images, and that similar scatterers may exist nearby within a pixel. In the future, we will derive a PDF that can explain conditions where similar scatterers are available within a SAR image pixel

\section{CONCLUSIONS}

We presented the PDFs of scatterings derived from fully polarimetric SAR images. We first introduced a theoretical PDF on the condition that image pixels have scatterers showing random backscattering. We then generated PDFs of the scatterings derived from data observed using concrete blocks with different orientation angles, and from an ALOS/PALSAR image. Analysis of the PDFs and the derived statistics reveal that the $P_{v+c}$ curves of the PDFs are smooth and most similar to the normal distribution among all the scatterings derived from fully polarimetric SAR images. We found that $T_{v+c}$ works most effectively because of its similarity to the normal distribution. We also found that PDFs derived from datasets are different from theoretical PDFs. This may be because the assumption that a pixel has random scatterers does not apply to actual images. In future, we will propose an improved method for estimating urban density that normalizes a scattering without using the mean and standard deviation.

\section{ACKNOWLEDGEMENTS}

The author thanks Y. Yamaguchi (Niigata University) and his students for offering experimental facilities and for their many helpful comments. This research was supported by a program of the Fourth Advanced Land Observing Satellite-2 Research Announcement (RA4), Japanese Aerospace Exploration Agency. ZmapTown II (ZENRIN) was provided by the Center for Spatial Information Science at the University of Tokyo.

\section{REFERENCES}

Esch, T., Marconcini, M., Felbier, A., Roth, A., Heldens, W. Huber, M. Schwinger, M., Taubenböck, H., Müller, A. and Dech, S., 2013. Urban footprint processor - fully automated processing chain generating settlement masks from global data of the TanDEM-X mission, IEEE Geosci. Remote Sens. Letters, 10, pp. 1617 - 1621.

Kajimoto M. and Susaki J., 2013a. Urban area extraction from polarimetric SAR images using polarization orientation angle. IEEE Geosci. Remote Sens. Letters, 10, pp. 337 - 341.

Kajimoto, M. and Susaki, J., 2013b. Urban density estimation from polarimetric SAR images based on a POA correction method. IEEE J. Selected Topics Applied Earth Observ. Remote Sens., 6(3), pp. $1418-1429$.

Niu, X. and Ban, Y., 2012. An adaptive contextual SEM algorithm for urban land cover mapping using multitemporal highresolution polarimetric SAR data. IEEE J. Selected Topics Applied Earth Observ. Remote Sens., 5, pp. 1129 - 1139.

Perissin, D. and Wang, T., 2012. Time-series InSAR applications over urban areas in China. IEEE J. Selected Topics Applied Earth Observ. Remote Sens., 4, pp. 92 - 100.

Susaki, J., Kajimoto, M. and Kishimoto, M., 2014. Urban density mapping of global megacities from polarimetric SAR images. Remote Sens. Environ., 155, pp. 334 - 348.

Yamaguchi, Y., Sato, A., Boerner, W., Sato, R. and Yamada, H., 2011. Four-component scattering power decomposition with rotation of coherency matrix. IEEE Trans. Geosci. Remote Sens., 49, pp. $2251-2258$. 